\title{
Effect of Saccadic Adaptation on Sequences of Saccades
}

\author{
Muriel Panouillères \\ ImpAct team, CRNL - INSERM U1028 - CNRS UMR5292 - Lyon University \\ Roméo Salemme \\ ImpAct team, CRNL - INSERM \\ U1028 - CNRS UMR5292 - Lyon \\ University \\ Christian Urquizar \\ ImpAct team, CRNL - INSERM \\ U1028 - CNRS UMR5292 - Lyon \\ University \\ Denis Pélisson \\ ImpAct team, CRNL - INSERM U1028 - CNRS UMR5292 - Lyon University
}

\begin{abstract}
Accuracy of saccadic eye movements is maintained thanks to adaptation mechanisms. The adaptive lengthening and shortening of reactive and voluntary saccades rely on partially separate neural substrates. Although in daily-life we mostly perform sequences of saccades, the effect of saccadic adaptation has been mainly evaluated on single saccades. Here, sequences of two saccades were recorded before and after adaptation of rightward saccades. In 4 separate sessions, reactive and voluntary saccades were adaptively shortened or lengthened. We found that the second saccade of the sequence always remained accurate and compensated for the adaptive changes of the first rightward saccade size. This finding suggests that adaptation loci are upstream of the site where the efference copy involved in sequence planning originates.
\end{abstract}

Keywords: Eye movements, Saccadic adaptation, Sequence of Saccades, Efference copy, Human

\section{Introduction}

Our movements allow us to interact with the surrounding world. The contribution of the motor system in acquisition of sensory information is particularly critical for vision. Because high visual acuity is restricted to the central part of the retina, saccadic eye movements are necessary for collecting detailed visual information. Thus, a series of different snapshots of the visual scene is provided by retinal input following each saccade. There is a large consensus about the brain capability to predict the retinal input sampled after each saccade, helping both visual exploration and the generation of subsequent saccades (Wurtz, 2008). Regarding saccade production, this prediction capability has been demonstrated in double-saccade tasks. These tasks have indeed shown that both monkey and human subjects can generate two accurate saccades in response to the rapid succession of two flashed targets, even when the second target disappears during the latency period of the first saccade (Hallett \& Lightstone, 1976; see review in Pisella et al., 2011). The accurate production of the second saccade without direct visual input relies on brain mechanisms that compensate for the eye displacement achieved during the first saccade and keep track of this nonvisual eye displacement information. In principle, this extra-retinal signal of eye displacement could be provided by ocular proprioceptive information or by a copy of the motor command sent to the extraocular muscles (termed efference copy or corollary discharge). However, empirical studies in the monkey have refuted the contribution of ocular proprioception (Guthrie, Porter, \& Sparks, 1983; Lewis, Zee, Hayman, \& Tamargo, 2001; Sparks \& Mays, 1983) and it is now widely accepted that the extra-retinal signals used to accurately plan subsequent saccades are based on an efference copy signal (Moschovakis, Scudder, \& Highstein, 1996; Robinson, 1975; Keller, 1981).

Saccade accuracy is also maintained over long time-scales thanks to adaptation mechanisms. These mechanisms induce progressive modifications of saccade amplitude and/or direction to compensate for enduring errors. In the laboratory, saccadic adaptation can be induced by systematically stepping the visual target during the saccade, generating an error between the saccade end position and the target position. By repeating this 
intra-saccadic target step in successive trials, saccade amplitude is progressively modified such as the eyes get closer to the stepped target position. Initially designed for reactive saccades, this doublestep target paradigm (McLaughlin, 1967) has been modified to study also adaptation of scanning voluntary saccades (Alahyane et al., 2007; Deubel, 1995). These saccades allow us to scan our environment and thus represent the most frequent category of saccades produced in daily life. Reactive saccades are less often produced and are aimed to track fast moving objects or reorient the eyes toward new stimuli that suddenly appear in our visual field. Recent studies showed that the adaptation of these two saccade categories depends on partially separate mechanisms (Alahyane et al., 2007; Collins \& Dore-Mazars, 2006; Cotti, Guillaume, Alahyane, Pelisson, \& Vercher, 2007; Cotti et al., 2009; Deubel, 1995; Erkelens \& Hulleman, 1993; Fujita, Amagai, Minakawa, \& Aoki, 2002; Panouilleres, Urquizar, Salemme, \& Pelisson, 2011; Zimmermann \& Lappe, 2009; Hopp \& Fuchs, 2002). Furthermore, saccades can be adaptively shortened (backward adaptation) or lengthened (forward adaptation) and evidence suggests that these two adaptations also rely on different processes (Catz, Dicke, \& Thier, 2008; Ethier, Zee, \& Shadmehr, 2008; Golla et al., 2008; Hernandez, Levitan, Banks, \& Schor, 2008; Kojima, Iwamoto, \& Yoshida, 2004; Panouilleres et al., 2009; Semmlow, Gauthier, \& Vercher, 1989; Zimmermann \& Lappe, 2010; Noto, Watanabe, \& Fuchs, 1999; Straube \& Deubel, 1995; Panouilleres et al., 2011).

Although in daily life, we mainly produce sequences of saccades, few studies have asked how they are affected by saccadic adaptation. It has been shown in monkey (Tanaka, 2003) and in human (Collins, 2010; Dore-Mazars, Vergilino-Perez, Collins, Bohacova, \& Beauvillain, 2006) that the second saccade of a sequence could compensate for the adaptive changes of the previous saccade. Indeed, the programming of second saccades took into account the adaptive change of the preceding saccade and their metrics were accordingly modified such as to remain accurately directed toward their target. This indicates that saccadic adaptation can take place above the site where the efference copy regulating saccade sequences originates. However, because of adaptation differences between species (Frens \& van Opstal, 1994; Straube, Fuchs, Usher, \& Robinson, 1997) and because the above human studies focussed on backward adaptation of reactive saccades (Collins,
2010; Dore-Mazars et al., 2006), it is still unknown whether a similar conclusion can be generalized to forward adaptation and to voluntary saccade in human. Therefore, the present study is aimed at evaluating the effect of the different types of saccadic adaptation on saccade sequences. Specifically, we wondered if the second saccade of a sequence would compensate for adaptive changes of the first saccade, and would then still be accurately directed toward its target. To address this question, the adaptive lengthening and shortening of reactive and voluntary rightward saccades were induced in separate sessions and, before and after each adaptation session, subjects performed sequences of two-saccades.

\section{Methods}

\section{Subjects}

Eight observers volunteered for the experiments (6 women, 6 naive subjects and authors MP and DP, mean age: $29.4 \pm 7.9$ years). All subjects had a normal or corrected to normal vision. The study conformed with the Code of Ethics of the World Medical Association (Declaration of Helsinki).

The study comprised four different experiments: adaptive shortening (backward adaptation) of reactive saccades, adaptive shortening of voluntary saccades, adaptive lengthening (forward adaptation) of reactive saccades and adaptive lengthening of voluntary saccades. Four participants performed all experiments, three subjects participated to three experiments and the last one was tested only in the voluntary saccade forward adaptation experiment. No subject performed the same experiment twice. When volunteers performed different experiments, the experiments' order was counterbalanced and at least 5 days separated any two experiments. Thus, in total, there were 6 repetitions of each backward experiment and 7 repetitions of each forward experiment.

\section{Apparatus}

The experiments took place in a dark room with the subjects seating $57 \mathrm{~cm}$ from a $140 \mathrm{~Hz}$ computer screen $\left(30^{\circ} \times 40^{\circ}\right)$ controlled by a Visual Stimuli Generation system (CRS Cambridge, UK). Head movements were restrained by chin and cheekbone rests. Visual targets were $0.6 \mathrm{~cm}$ diameter black disks presented on a grey background. Vertical and horizontal positions of both eyes were monitored at $250 \mathrm{~Hz}$ using an infra-red eye tracker (Eyelink II, SR Research, Canada). The eye tracker was 
calibrated before each experiment by asking the subjects to fixate 9 targets constituting a $28^{\circ} \times 38^{\circ}$ rectangle. Software developed in the laboratory allowed the monitoring of eye movement data both for off-line analysis and for on-line modification of the visual display during primary saccades.

\section{Experimental design}

Each experiment comprised three phases: preadaptation, adaptation and post-adaptation.

\section{Adaptation phase}

Adaptation of rightward reactive saccades was induced using the double-step target paradigm (McLaughlin, 1967) (example of backward adaptation in Figure 1A). At the beginning of a trial, the subjects gazed at a central fixation point (FP). After a random delay of 1600,1800 or 2000 $\mathrm{ms}$, FP was turned off and simultaneously a target appeared at $+8^{\circ}$ (right). When the ocular velocity reached a $\sim 90^{\circ} / \mathrm{sec}$ threshold (ranging from 85 to $95 \%$ sec between subjects), the target stepped to a new position. This intra-saccadic step corresponded to $25 \%$ of the initial target eccentricity for the first 2 blocks of 24 trials (blocks called a25 and b25), to $30 \%$ for the following 24 trials (c30), to $35 \%$ for the next 24 trials (d35) and to $40 \%$ for the remaining 24 trials (e40). In different experiments, the intra-saccadic target step was directed either toward the fixation point to induce backward adaptation (Figure 1A) or away from the fixation point to produce forward adaptation.

A modified version of the double-step target paradigm (Alahyane et al., 2007; Deubel, 1995) was used to adapt rightward voluntary saccades (example of backward adaptation in Figure 1B). At the beginning of a trial, subjects looked at a FP $4^{\circ}$ above the horizontal meridian. Then, $1600 \mathrm{~ms}$ later, a circle appeared around the FP simultaneously with 2 targets: one located $4^{\circ}$ below the FP (screen centre) and the other one located at $+8^{\circ}$ (right) on the horizontal meridian. After $500 \mathrm{~ms}$, the circle disappeared, signalling the subject to make first a vertical saccade to the target below the FP and then a second, horizontal, saccade to the lateral target. During the horizontal voluntary saccade (velocity threshold $90^{\circ} / \mathrm{sec}$ ), the full set of targets stepped horizontally either toward the fixation point to elicit backward adaptation (Figure 1B) or away from the fixation point to elicit forward adaptation. The number and size of intra-saccadic target steps were identical to those used for the reactive saccade adaptation.

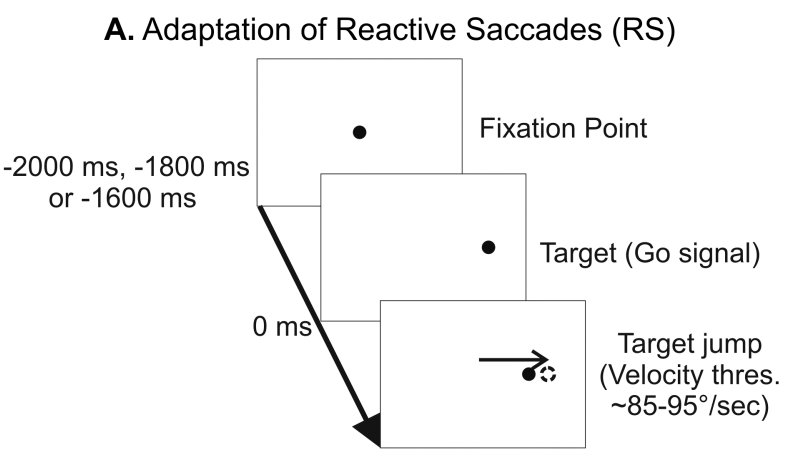

B. Adaptation of Voluntary saccades (VS)

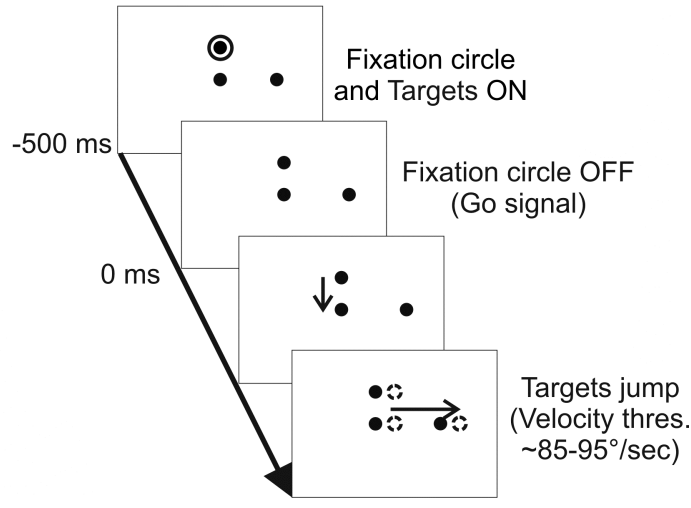

Figure 1: Backward adaptation protocol of reactive (A) and voluntary $(B)$ saccades. Arrows represent primary saccades. When a horizontal primary saccade is detected, all targets jumped in direction of the centre of the screen to induce a progressive decrease of saccadic gain. To elicit an increase of gain (forward adaptation protocol not shown), all targets jumped away from the centre of the screen.

\section{$\underline{\text { Pre- and post-adaptation phases }}$}

In each pre- and post-adaptation phase, participants performed two variations of a saccade sequence task, called refixation and double-target tasks respectively. The common goal of these tasks was to test if the adaptive modification of the amplitude of the first saccade of a sequence would be compensated for by the second saccade. The first saccades were always horizontal in these tasks. The second saccades were horizontal and directed toward the fixation point position in the refixation task, whereas they could be vertical, oblique or horizontal in the double-target task. This second task allowed us to evaluate if the adaptive change of the first saccade could differently affect these second saccades according to their vector. In preand post-adaptation, two-saccade sequences could start either with a first rightward saccade ("rightward trials"), or with a first leftward saccade ("leftward trials"). No adaptive changes of the first saccades were expected in the leftward trials (Albano, 1996; Deubel, Wolf, \& Hauske, 1986; Frens \& van Opstal, 1994; Miller, Anstis, \& 
Templeton, 1981), so these trials were aimed to measure the level of compensation by the second saccade of the first saccade natural variability. This will be used as a baseline for the measure of the compensation in the rightward trials.

In the refixation task, the subjects were instructed to make a first saccade toward a target presented at $\pm 8^{\circ}$ and then to look back as precisely as possible to the start position before reappearance of the fixation point. All 24 trials began with a central fixation point (FP) presented for 1600,1800 or $2000 \mathrm{~ms}$. Simultaneously with the target appearance, FP was turned off. Then, the target was switched off when the eye velocity reached a threshold of $\sim 90 \% \mathrm{sec}$. After the end of this first saccade, the participants had to make a second saccade toward the remembered location of FP. Rightward and leftward trials (target presented at $+8^{\circ}$ and $-8^{\circ}$, respectively) were randomly interleaved.

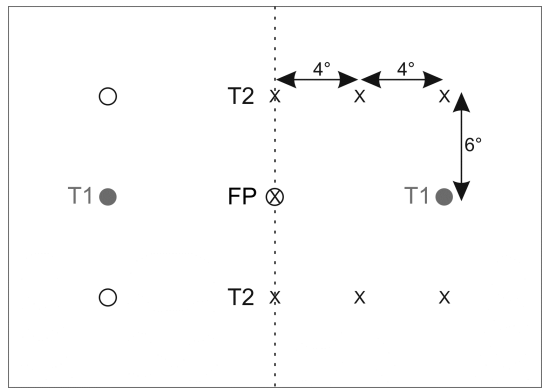

Figure 2: Positions of the $I^{\text {st }}$ (T1) and $2^{\text {th }}$ (T2) targets of the double-target task. The gray dots indicate the locations of the $1^{\text {st }}$ target whereas the black symbols (' $x$ ' and open circle) represent the positions of the $2^{\text {nd }}$ target (one such position corresponded to that of the fixation point). In the case of rightward trials, the $2^{\text {nd }}$ target (' $x$ ' symbols) could appear at 6 different locations on the right part of the screen or at the central location. In the case of leftward trials, the $2^{\text {nd }}$ target (open circles) could appear either at one of the 2 locations shown on the left part of the screen or at the central screen location.

In the double-target task, observers were instructed to produce two accurate saccades towards the remembered location of two flashed targets. All 80 trials of this task began with a central fixation period of 2100,2300 or $2500 \mathrm{~ms}$. The first target (T1) was briefly presented at $\pm 8^{\circ}$ for $120 \mathrm{~ms}$ and the second target (T2) could be presented at different locations for $70 \mathrm{~ms}$. When $\mathrm{T} 1$ was presented at $+8^{\circ}$ (rightward trials), T2 could appear at one of seven different locations (Figure 2 ). When $\mathrm{T} 1$ was presented at $-8^{\circ}$ (leftward trials), T2 was displayed at one of three locations $\left(6^{\circ}\right.$ above or below the first target and at the fixation point). Subjects were asked to wait until the disappearance of $\mathrm{T} 2$ before initiating the first saccade, in order to avoid peri-saccadic compression.

\section{Data analysis}

\section{$\underline{\text { Saccade parameters }}$}

Movements of both eyes were analyzed off-line with a custom program developed in the Matlab v.7.1 environment (Mathworks, MA., U.S.A.). The position and time of the beginning and end of all saccades were detected on the basis of a velocity threshold of $50 \% \mathrm{sec}$ and were extracted from the mean data of the right and left eye. For the adaptation phase, these parameters were identified only for the horizontal saccades. For the pre- and post-adaptation phases, 2 saccades were detected for each trial: the 2 horizontal saccades respectively directed toward $\mathrm{T} 1$ and remembered FP in the refixation task and, in the double-target task, the first horizontal saccade toward $\mathrm{T} 1$ and the second (vertical, oblique or horizontal) saccade toward T2. Saccade latency was calculated in pre-adaptation as follows: $1^{\text {st }}$ saccade latency was the delay between the $1^{\text {st }}$ target appearance and the beginning of the $1^{\text {st }}$ saccade and the $2^{\text {nd }}$ saccade latency corresponded to the fixation time between the $1^{\text {st }}$ and the $2^{\text {nd }}$ saccade. Saccade latency in the first block of the adaptation phase was computed for reactive saccades as the time between target appearance and beginning of eye movements and for voluntary saccades as the period between the end of the vertical saccade and the start of the horizontal voluntary saccade. Saccades contaminated by a blink were eliminated $(2.7 \pm 2.4 \%$ of total trials $)$.

Saccade gain was calculated as the ratio between primary saccade amplitude and retinal error (distance between target and initial eye position). For the adaptation phase, saccadic gain change was calculated for each trial using the mean gain of the first five adaptation trials as baseline. Note that this calculation could slightly underestimate the amount of adaptation, because some change of saccade gain is already expected in the first five adaptation trials. However, the main objective of the present study was to compare adaptation and transfer between different conditions, a comparative analysis not affected by this general underestimation. Gain change was computed in such a way that positive values indicated expected modifications according to the adaptation condition: a decrease of gain for backward adaptation and an increase of gain for forward adaptation. This allowed a more direct comparison of adaptation between the backward and forward protocols. For the refixation and the 
double-target tasks, the gain change of the first rightward saccade in post-adaptation relative to preadaptation was computed as a measure of adaptation transfer. In the refixation task, we also measured the gain change of the second rightward saccade of the leftward trials, as another measure of adaptation transfer, this time when the second saccade of the sequence was directed toward the adapted field.

To test if modifications of the first rightward saccade were compensated for by the second saccade, two parameters describing this last saccade's starting and end positions were calculated. First, we computed the change of initial position of each second saccade in post-adaptation relative to the corresponding mean value in preadaptation trials. This parameter reflects the adaptive modification of the first saccade in rightward trials and the natural variability of this same saccade in leftward trials, taking also in consideration the production of corrective saccades between first and second saccades. Second, we calculated the modification of horizontal amplitude of the second saccades in post-adaptation relative to the corresponding mean value in pre-adaptation trials. Lastly, we plotted the relationship between these two parameters of second saccades (i.e. changes of horizontal amplitude as a function of modifications of initial position). A negative correlation between these two parameters will reveal the existence of compensation for the adaptive change or natural variation of the first saccade amplitude (Figure 3). Conversely, a lack of correlation between these two parameters will indicate an absence of compensation (Figure 3). These correlations were computed for each subject of each experiment, separately for the refixation and the double-target tasks.

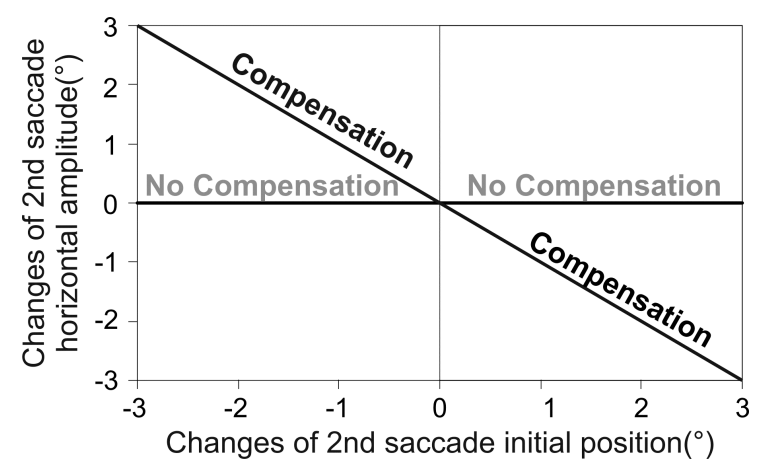

Figure 3: Predicted correlations between the changes of $2^{\text {thd }}$ saccade horizontal amplitude and the changes of $2^{\text {id }}$ saccade initial position in post-adaptation relative to pre-adaptation.

\section{$\underline{\text { Statistical analysis }}$}

Statistical analyses were performed with the STATISTICA 9 software package. An ANOVA with "blocks of trials" (a25 versus b25 versus ... e40), "saccade type" (reactive versus voluntary) and "adaptation direction" (backward versus forward) was performed on the gain changes calculated during the adaptation phase. Then, to check if the adaptation modified the $1^{\text {st }}$ rightward saccade of the two-saccade tasks, the gain of these saccades was compared between pre- and postadaptation with t-tests, separately for the four experiments and for the refixation and double-target tasks. Two two-way ANOVAs were performed separately for the refixation and the double-target tasks, to detect an effect of the "saccade type" (reactive versus voluntary) and/or of the "adaptation direction" (backward versus forward) factors on the mean gain changes of the $1^{\text {st }}$ rightward saccades. In the refixation task, we compared the gain between pre- and postadaptation of the $2^{\text {nd }}$ rightward saccade of the leftward trials using t-tests.

The level of compensation of the second saccades for the changes of the first saccades was evaluated by the slope of the correlation of the compensation relationship described above (see section: Saccade parameters). One-way ANOVAs testing the "T2 position" factor were performed on the compensation slopes of the double-target task separately for the 4 adaptation experiments. No effect of this factor was detected $(F[2,6]<2.28$; $p>0.18$ ), showing that the compensation did not depend on the second saccade vector. Therefore, for the following analyses and separately for the four experiments, new correlations were calculated after pooling all T2 positions of the double-target task. An ANOVA with the "saccade type", the "adaptation direction", the "first saccade direction" (rightward versus leftward) and the "task" (refixation versus double-target) factors was performed on the slope of all compensation correlations. The compensation slopes were compared to -1 (full compensation hypothesis) and to 0 (no compensation hypothesis) in separate $t$ tests for refixation and double-target tasks.

Error bars in the different figures are SEs. Statistical significance was set at $\mathrm{p}<0.05$. 


\section{Results}

The main aim of this study was to determine if the adaptive changes of a saccade could be compensated for by the subsequent saccade. Reactive and voluntary saccades were adaptively shortened and lengthened in four different experiments and sequences of two saccades were performed before and after these adaptations. First, the gain changes of rightward saccades during the adaptation phase will be evaluated. Second, the transfer of these adaptive changes to the $1^{\text {st }}$ rightward saccade of the two sequence tasks will be examined, as well as the transfer to the $2^{\text {nd }}$ rightward saccades of the leftward trials for the refixation task. Lastly, the compensation of the first saccade adaptive changes by the $2^{\text {nd }}$ saccade will be described.

\section{Time-course of adaptation}

Reactive and voluntary saccades were adapted in different experiments. Mean saccadic latency during the first adaptation block was $227 \pm 10 \mathrm{~ms}$ for reactive saccades and $428 \pm 30 \mathrm{~ms}$ for voluntary saccades. The significant difference between these two latencies (unpaired t-tests, $\mathrm{p}<0.001$ ) implies that our two adaptation paradigms were efficient in triggering reactive and voluntary saccades.

The time-course of gain changes during the adaptation phase relative to the first five trials is presented in Figure 4A for reactive saccades and in Figure 4B for voluntary saccades. Positive gain changes indicated that for both saccade types, saccade gain decreased across blocks of trials for backward adaptation whereas it increased for forward adaptation. These progressive gain changes were revealed by a significant effect of the "block of trials" factor $(F[4,110]=21.4, p<0.001)$. An effect of the "adaptation direction" factor revealed larger gain changes for backward adaptation than for forward adaptation $(F[1,110]=13, p<0.001)$. The absence of "saccade type" factor effect indicates that similar adaptation was induced for both saccade types $(\mathrm{F}[1,110]=0.48, \mathrm{p}=0.83)$.

Thus, the intra-saccadic target step induced a similar gain modification of rightward reactive and voluntary saccades, but these gain changes were stronger for backward adaptation than for forward one.

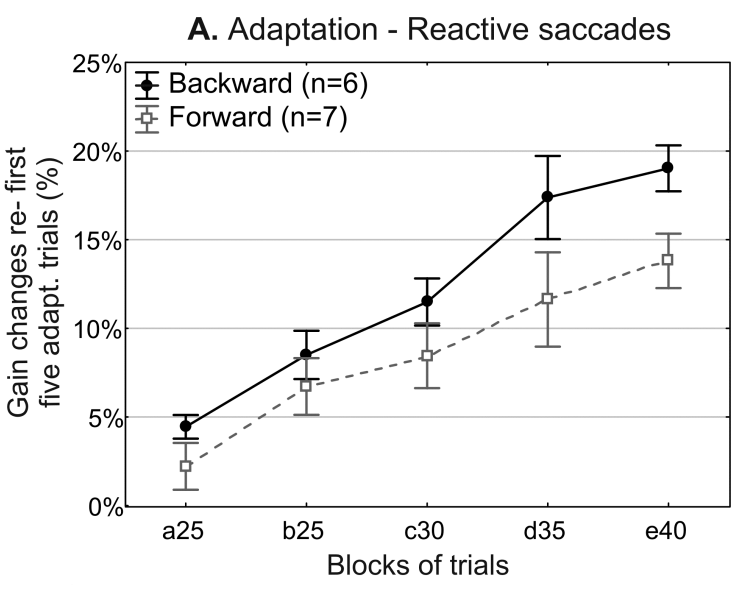

B. Adaptation - Voluntary saccades

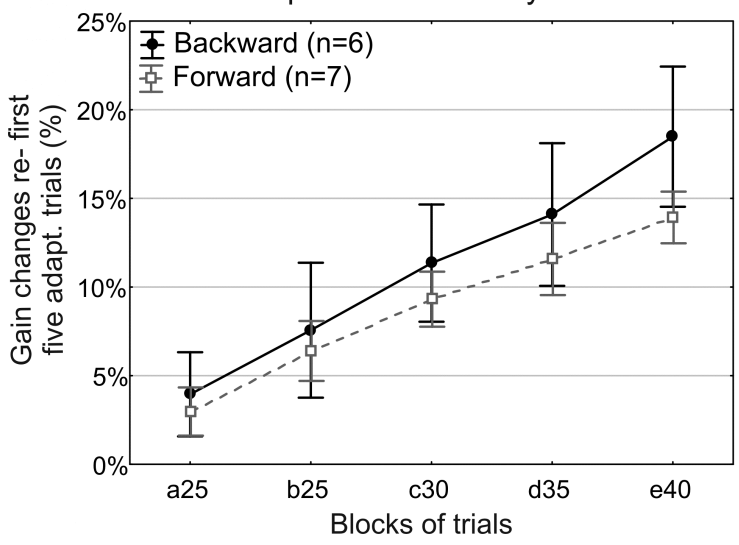

Figure 4: Time-course of saccadic gain during adaptation of reactive $(A)$ and voluntary (B) saccades. Mean gain changes relative to the first five trials of adaptation are represented as a function of the blocks of trials (a25, b25, c30, ..., e40) of the adaptation phase. Backward ( $\bullet$ ) and forward () adaptation are shown superimposed.

\section{Transfer of adaptation to rightward saccades of the two-saccade tasks}

\section{Refixation task}

In the refixation task, first saccades had a mean latency of $277 \pm 46 \mathrm{~ms}$ and second saccades presented a mean latency of $543 \pm 198 \mathrm{~ms}$. In all 12 repetitions of the backward experiments a significant gain decrease of the first rightward saccades was observed after adaptation (unpaired $\mathrm{t}$ tests, $\mathrm{p}<0.05$ ). Across repetitions, the gain decrease averaged $17.7 \pm 1.9 \%$ for reactive saccades and 10.6 $\pm 1.7 \%$ for voluntary saccades (Figure $5 \mathrm{~A}$ ) and was significant (paired $\mathrm{t}$ tests, $\mathrm{p}<0.001$ and $\mathrm{p}<0.01$ respectively). Within the 14 repetitions of the forward experiments, 2 did not reveal any significant gain increase of the first rightward saccade (unpaired t tests, $p>0.05$ ) and the results of these 2 repetitions were discarded for all the following analyses. Across the remaining 12 repetitions, the gain of the first rightward saccades 
significantly increased by $13.6 \pm 2.3 \%$ after reactive saccades adaptation and by $11.3 \pm 1.9 \%$ after voluntary saccades adaptation (Figure $5 \mathrm{~A}$ - paired $\mathrm{t}$ tests, $\mathrm{p}<0.01)$. As expected, the gain of the first leftward saccades remained unchanged in all experiments (Table 1), in agreement with the known directional selectivity of saccadic adaptation (Albano, 1996; Deubel et al., 1986; Frens \& van Opstal, 1994; Miller et al., 1981).

\section{A. Refixation task - first rightward saccades}

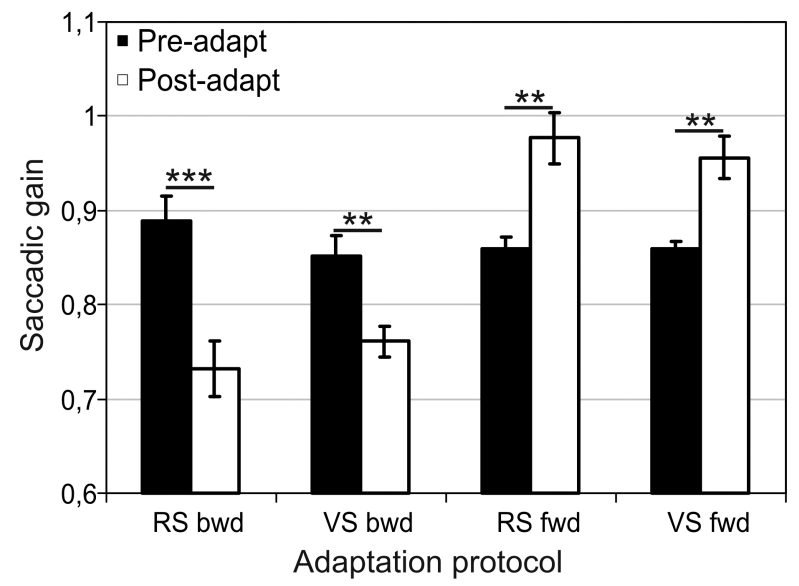

B. Double-target task - first rightward saccades

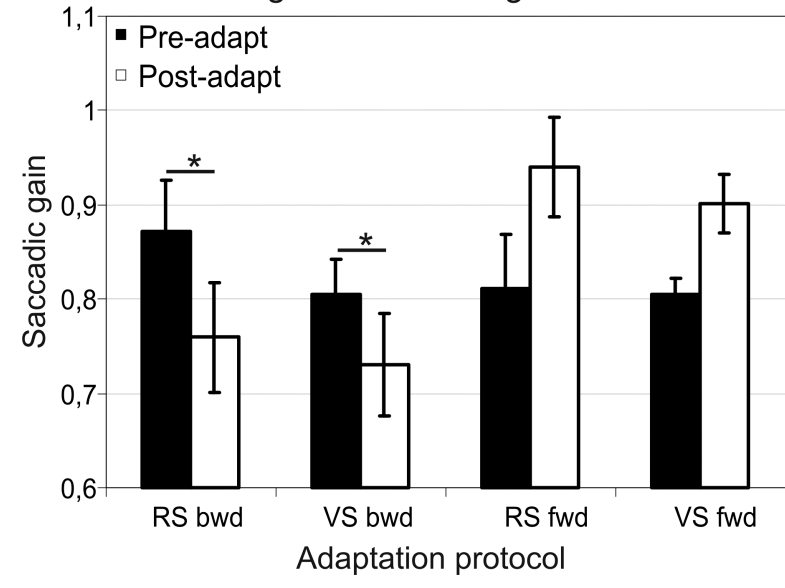

Figure 5: Gain changes of rightward saccades between the pre-and post-adaptation phases. Mean gains of the $I^{s t}$ rightward saccades of the refixation task (A) and of the double-target task (B) are shown in pre- and postadaptation separately for the backward adaptation of reactive saccades (RS bwd), the backward adaptation of voluntary saccades (VS bwd), the forward adaptation of reactive saccades (RS fwd) and the forward adaptation of voluntary saccades (VS fwd). Paired t-tests were used at the group level to measure significant gain modifications in post-adaptation relative to pre-adaptation: $* p<0.05$, $* * p<0.01$ and $* * * p<0.001$.

An ANOVA highlighted a main effect of the "saccade type" factor on the gain changes of the $1^{\text {st }}$ rightward saccades $(\mathrm{F}[1,20]=5.33 ; \mathrm{p}<0.05)$, but no effect of the "adaptation direction" factor, nor any interaction $(\mathrm{F}[1,20]<1.40, \mathrm{p}>0.24)$. This effect arose mainly from a stronger gain modification of rightward saccades after backward adaptation of reactive saccades than of voluntary ones (post-hoc Fisher's LSD test, $\mathrm{p}<0.05$ ). This difference can be explained by the fact that the adaptation of voluntary saccades does not transfer completely to reactive saccades (Alahyane et al., 2007; Collins \& Dore-Mazars, 2006; Cotti, Guillaume, Alahyane, Pelisson, \& Vercher, 2007; Deubel, 1995; Erkelens \& Hulleman, 1993; Fujita, Amagai, Minakawa, \& Aoki, 2002; Zimmermann \& Lappe, 2009), and that $1^{\text {st }}$ saccades of the refixation task are reactive (as indicated by their short latency).

In the leftward trials of the refixation task, the second saccades were directed rightward, i.e. in the adapted direction. Thus, we tested if adaptation transferred to these second rightward saccades. For the backward experiments, a significant reduction of gain $(11 \pm 2.6 \%)$ was observed for one repetition after adaptation of reactive saccades and for three repetitions (mean: $10 \pm 2.4 \%$ ) after adaptation of voluntary saccades (unpaired t tests, $\mathrm{p}<0.05$ ). A non significant gain reduction was observed in all other repetitions. In the forward experiment, a statistical increase of gain $(9 \pm 2.2 \%)$ was observed for one repetition after adaptation of reactive saccades (unpaired t test, $\mathrm{p}<0.01$ ). A non-significant increase of gain was found in five repetitions and a stable or slightly decreased gain in the last 6 repetitions.

As an intermediate conclusion, all four different adaptations strongly and reliably transferred to the first rightward saccades of the refixation task (except for 2 repetitions of the forward experiments, discarded for the following analyses). Contrarily, concerning the second rightward saccades of this task, the transfer was weak for all adaptation types.

Table 1

Gain of the first leftward saccades in pre- and postadaptation of the refixation task

\begin{tabular}{cccc}
\hline Protocol & Pre- & Post- & P-values \\
\hline RS backward & $0.88 \pm 0.03$ & $0.86 \pm 0.04$ & $\mathrm{p}=0.47$ \\
RS forward & $0.89 \pm 0.05$ & $0.88 \pm 0.06$ & $\mathrm{p}=0.30$ \\
VS backward & $0.86 \pm 0.03$ & $0.87 \pm 0.03$ & $\mathrm{p}=0.62$ \\
VS forward & $0.88 \pm 0.01$ & $0.90 \pm 0.02$ & $\mathrm{p}=0.13$
\end{tabular}

Note. Paired t-tests compared the individual mean gain in pre- and post-adaptation. 


\section{Double-target task}

In this task, the mean saccadic latency was 487 $\pm 94 \mathrm{~ms}$ for first saccades and $510 \pm 147 \mathrm{~ms}$ for second saccades. In the backward adaptation condition, reactive saccades adaptation was tested 6 times and voluntary saccades adaptation tested in 6 other repetitions. In both cases, only 5 out of 6 repetitions revealed a significant shortening of the first rightward saccades in the double-target task (unpaired $t$ test, $\mathrm{p}<0.05$ ). As for the refixation task, the two repetitions without significant transfer were discarded from further analysis. Across all remaining repetitions, the gain of first rightward saccades decreased by $13.0 \pm 3.4 \%$ and by 9.8 $\pm 3.4 \%$ after reactive and voluntary saccade adaptation, respectively (Figure 5B - paired t-test, $\mathrm{p}<0.05)$. In the forward condition, only 5 out of 7 repetitions revealed a significant increase of $1^{\text {st }}$ rightward saccades gain after adaptation of reactive saccades and only 3 out of 7 disclosed a significant transfer of adaptation after voluntary saccades adaptation. After excluding the repetitions without significant transfer, the average gain increase across the remaining repetitions was $17.3 \pm 8.5 \%$ and 11.9 $\pm 4.0 \%$ after adaptation of reactive and voluntary saccades respectively (Figure 5B). Because of the small number of repetitions, these changes only approached significance level (paired t test, $\mathrm{p}=0.06$ for reactive saccades adaptation and $\mathrm{p}=0.08$ for voluntary saccades adaptation).

As expected, when the first saccades were performed in the non-adapted direction (leftward trials), the gain was not modified in postadaptation, except after forward adaptation of voluntary saccades where a significant increase of gain was detected (Table 2 , paired t test, $\mathrm{p}=0.02$ ).

Table 2

Gain of the first leftward saccades in pre- and postadaptation of the double-target task

\begin{tabular}{cccc}
\hline Protocol & Pre- & Post- & P-values \\
\hline RS backward & $0.82 \pm 0.06$ & $0.84 \pm 0.08$ & $\mathrm{p}=0.41$ \\
RS forward & $0.86 \pm 0.09$ & $0.90 \pm 0.09$ & $\mathrm{p}=0.10$ \\
VS backward & $0.80 \pm 0.05$ & $0.78 \pm 0.05$ & $\mathrm{p}=0.52$ \\
VS forward & $0.88 \pm 0.03$ & $0.95 \pm 0.04$ & $\mathbf{p}=\mathbf{0 . 0 2}$ \\
\hline
\end{tabular}

Note. Paired t-tests compared the individual mean gain in pre-and post-adaptation.

Then, an ANOVA with the "saccade type" and "adaptation direction" factors was performed on gain changes in post-adaptation relative to pre- adaptation for the first rightward saccades. No effect was found for this ANOVA $(F[1,14]=0.57$; $\mathrm{p}>0.46$ ), suggesting that similar modifications of gain were achieved in the double-target task whatever the adaptation direction or the type of adapted saccade.

In conclusion, for both the refixation and double-target tasks, in most cases a significant modification of gain was observed for the first saccades performed in the adapted direction, and cases in which this did not occur were more frequent after forward than backward adaptation (8 and 2 times, respectively). The following analyses, which address our main objectives, are based on the repetitions that did show a significant effect of adaptation on their first rightward saccades.

\section{Compensation of the adaptive changes of the first saccade by the second saccade}

As described in Methods, compensation in the two-saccade tasks was evaluated by first computing two parameters of the second saccades (changes of initial position and of horizontal amplitude between pre- and post-adaptation) and then by plotting the relationship between these two parameters. Representative examples of this relationship, drawn from one subject in the case of the refixation task, are plotted in Figure 6 . When preceded by a rightward saccade, the second saccade initial position is displaced toward the fixation point after backward adaptation (Figure 6A - negative values) or away from the fixation point after forward adaptation (Figure 6B - positive values). For both adaptation directions (backward and forward), there is a negative correlation between the amplitude and starting location of the second saccade (Figure 6A and $6 \mathrm{~B}$, black lines). Note that similar correlations are observed when the first saccade is directed leftward, i.e. to the unadapted side (Figure 6, grey lines).

Similar plots were built for the double-target task but separately for the different horizontal positions of second target (not shown). Because there was no significant difference of the "T2 position" factor on the slope of these correlations (see Methods), new plots were built after pooling all T2 positions. The slope of these correlations, did not differ according to any of the following factors: the adapted saccade type, the adaptation direction, the first saccade direction (rightward versus leftward) or the task (four-way ANOVA -see Methods-, $F[1,64]<2.01 ; \mathrm{p}>0.16$ ). This leads to several conclusions. First, the compensation of the adaptation by the second saccade was similar 
regardless of the direction of adaptation and of the type of adapted saccade. Second, this compensation did not depend on the task (refixation or doubletarget). Third, the compensation by the second saccade was similar when it took place after an adapted saccade or a non-adapted one, suggesting that the adaptation did not modify the compensation of natural saccade variability.

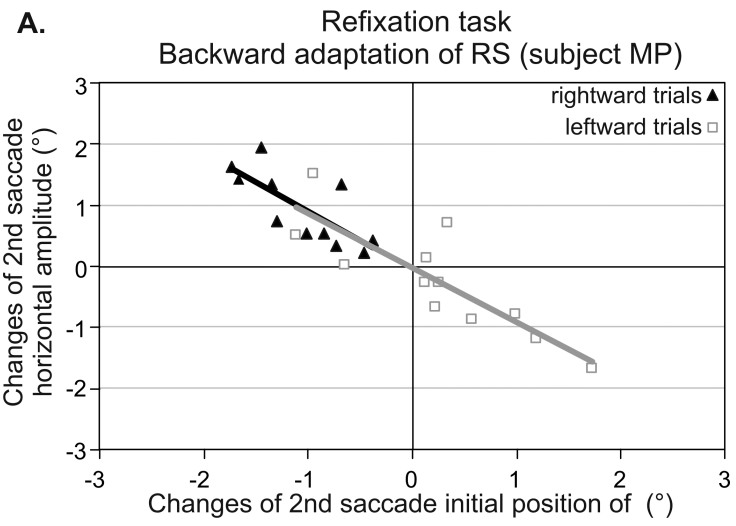

B.

Refixation task

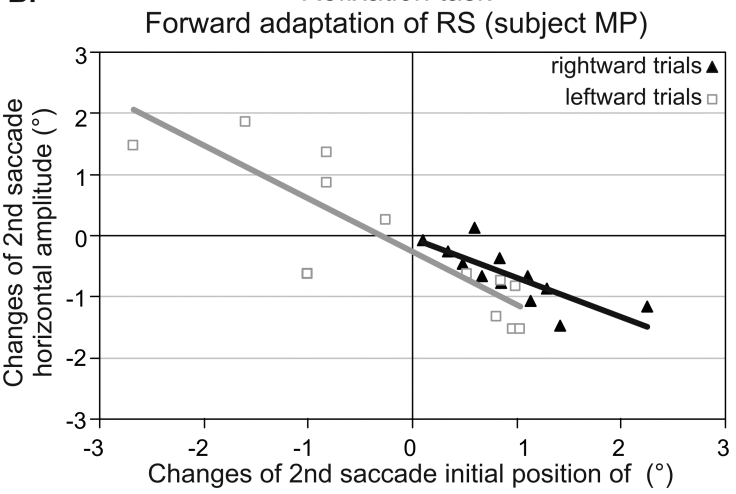

Figure 6: Modifications of the second saccade of the refixation task after backward (A) and forward (B) adaptation: example of a representative subject (MP). Changes of $2^{\text {nd }}$ saccade horizontal amplitude in postadaptation relative to pre-adaptation are correlated to changes in $2^{\text {nd }}$ saccade initial position. The correlations were computed separately for the cases where the $I^{\text {st }}$ saccade was performed in the adapted direction in rightward trials (4) or in the non-adapted direction in the leftward trials ().

To statistically evaluate how much the second saccades compensated for changes of the first saccades, the slopes of the previously described correlations were compared to -1 and to 0 (Figure $7 \mathrm{~A}$ and $7 \mathrm{~B}$ ). We found that, regardless of the first saccade direction (rightward versus leftward), the task, the adaptation direction and the type of adapted saccade, the compensation slope never differed from -1 (except for non-adapted saccade in the double-step task after backward adaptation of voluntary saccades, $\mathrm{p}<0.01$ ). In contrast, the compensation slope statistically differed from 0 in all cases, except for the double-target task after forward adaptation of voluntary saccades (saccades in the adapted direction), and for the refixation task after backward adaptation of reactive and of voluntary saccades (saccades in the non-adapted direction).

A. Refixation task - Compensation of 1st saccade

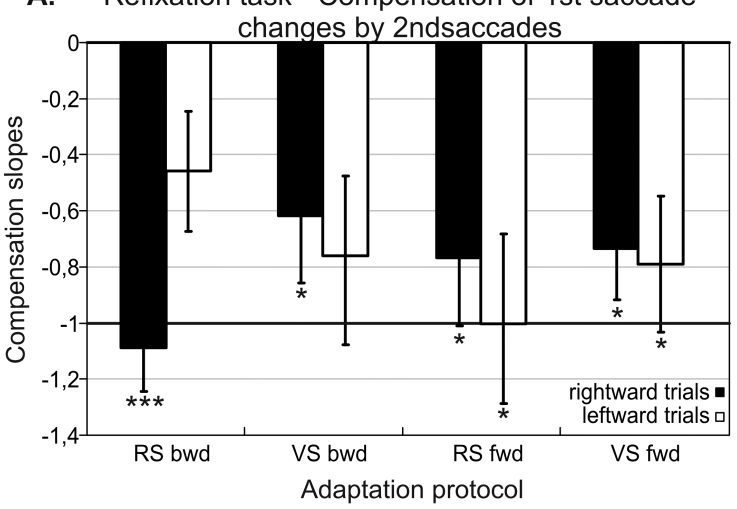

B. Double-target task - Compensation of 1st saccade

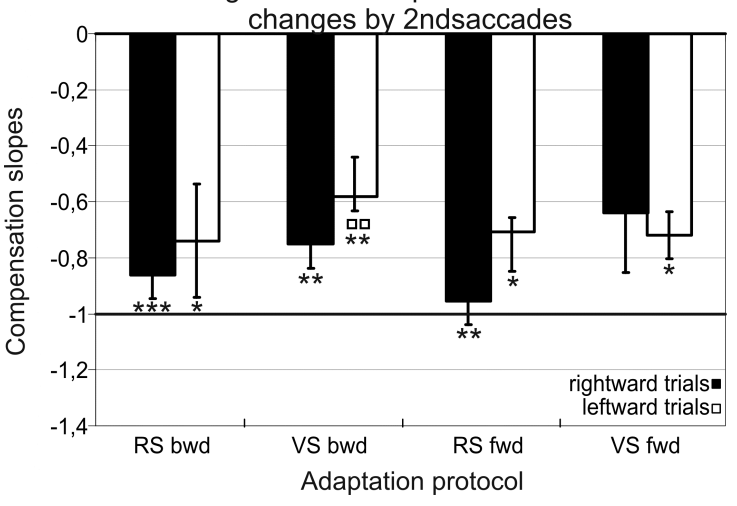

Figure 7: Compensation slopes for the refixation (A) and double-target (B) tasks. The slopes were calculated from the correlation between the changes of second saccade horizontal amplitude and the changes of second saccade initial position (example in Figure 7), separately for backward and forward adaptation of reactive and voluntary saccades. Significant differences with 0 (no compensation) are represented as: * $p<0.05, * * p<0.01$ and $* * * p<0.001$ and the significant difference with -1 (total compensation) is indicated by $p p p<0.01$ (t tests).

To conclude, the compensation of the adaptation by the second saccade was strong, even full in many cases, and similar regardless of the adaptation direction and of the adapted saccade type. Moreover, the compensation by the second saccade was similar whether the first saccade was adaptively modified or not.

\section{Discussion}

The main goal of the present study was to determine if the adaptive changes of the first saccade of a sequence affect the accuracy of the 
subsequent saccade. The effects of adaptive lengthening and shortening of reactive and voluntary saccades, known to rely on partially separate mechanisms (Pelisson, Alahyane, Panouilleres, \& Tilikete, 2010), were tested in four separate sessions. We found that in both saccade sequence tasks used in the present study, the second saccade fully compensated for the adaptive changes of first saccade, irrespective of the adaptation direction (backward or forward) and saccade category (reactive or voluntary). A similar compensation was also observed when the amplitude modifications of the first saccade resulted from natural fluctuation.

\section{Differences between the four adaptation mechanisms}

Differences between reactive and voluntary saccades adaptation are usually reported when adapting one category of saccades and testing its transfer to the other category (Alahyane et al., 2007; Collins \& Dore-Mazars, 2006; Cotti et al., 2007; Zimmermann \& Lappe, 2009). In agreement with these studies, we found that the backward adaptation of voluntary saccades transferred less, as compared to reactive saccades, to the first rightward saccades of the sequence in the refixation task.

Regarding backward and forward adaptations, considerable evidence have shown that the saccade adaptive lengthening has a slower time-course and induces smaller gain modifications than the adaptive shortening (Miller et al., 1981; Noto et al., 1999; Panouilleres et al., 2009; Semmlow et al., 1989; Straube et al., 1997; Zimmermann \& Lappe, 2010; Straube \& Deubel, 1995). In the present study, we also found a slower time-course of forward adaptation relative to backward adaptation. Moreover, we found that the adaptation transfer is less consistent after forward adaptation than after backward adaptation. Indeed, for the first rightward saccades of the two-saccade tasks, in 8 out of 14 repetitions a significant increase of gain was not observed after forward adaptation whereas a lack of significant decrease of gain after backward adaptation was found in only 2 out of 12 repetitions. Similarly, for the second rightward saccades (leftward trials of the refixation task), we did find a significant increase of gain after forward adaptation in only 1 out of 12 repetitions, whereas a significant decrease of gain after backward adaptation was found in 4 out of 12 repetitions.

To conclude, the classical dissimilarities between backward and forward adaptations (time- course, transfer to first saccades) were confirmed in the present study.

\section{Compensation of the adaptive changes of $a$ saccade by the subsequent saccade}

The main objective of this study was to assess whether a saccade can compensate for the adaptive changes of the previously performed saccade and thus, still be accurately directed toward its target. We found full compensation when reactive and voluntary saccades were adaptively shortened or lengthened. These results are in agreement with monkey data for backward and forward adaptation of reactive saccades (Tanaka, 2003) and with human data for backward adaptation of reactive saccades (Collins, 2010; Dore-Mazars et al., 2006).

The novelty of our study is to demonstrate a full compensation for all four different adaptation conditions (shortening and lengthening of reactive and voluntary saccades). As mentioned in the introduction, extra-retinal signals encoding eye position after the first saccade are necessary to produce a subsequent accurate saccade. These extra-retinal signals are mainly provided by an efference copy of oculomotor commands originating upstream from motoneurons (Guthrie et al., 1983; Mays \& Sparks, 1980; Schiller \& Sandell, 1983; Sparks \& Mays, 1983; Sparks, Mays, \& Porter, 1987; Steinbach, 1987). Our results, showing that full compensation still occurs after all types of adaptation (backward and forward, of reactive and voluntary saccades), therefore suggest that the saccadic adaptation sites are located upstream from the site where the efference copy originates. Because backward adaptation of reactive saccades is supposed to take place at a low level of sensory-motor transformation (Alahyane et al., 2007; Collins \& Dore-Mazars, 2006; Cotti et al., 2007; Cotti et al., 2009; Panouilleres et al., 2009), the efference copy necessary for accurate saccade programming in saccade sequences must also originate at a further downstream -or peripherallevel.

What could be the level where the efference copy originates? Recent studies starting to unravel the neural substrates of eye efference copy signals have notably found an involvement of the superior colliculus / thalamus / frontal cortex pathway (Gaymard, Rivaud, \& Pierrot-Deseilligny, 1994; Ostendorf, Liebermann, \& Ploner, 2010; Sommer \& Wurtz, 2008). Note that thalamic lesions supposed to alter this pathway only partially impair saccadic compensation, suggesting that other, as yet unknown pathways, are also involved (Sommer \& 
Wurtz, 2008). Combined with current hypotheses on the motor level of saccadic adaptation, notably backward adaptation of reactive saccades (see for reviews: Iwamoto \& Kaku, 2010; Pelisson et al., 2010), we speculate that the eye efference copy signal could originate either at the superior colliculus level or at level further downstream.

\section{Conclusion}

Although the adaptive lengthening and shortening of reactive and voluntary saccades rely on partially separate mechanisms, we found that when a saccade was adaptively modified, the subsequent saccade fully compensated for these adaptive changes and was then still accurately directed toward its target. This means that in human all four saccadic adaptation mechanisms take place upstream from the site where the efference copy is encoded.

\section{Acknowledgments}

We thank all the subjects for their kind participation. Experiments were performed in the "Mouvement et Handicap" Institut Fédératif de Neurosciences de Lyon platform (Lyon, France).

\section{References}

Alahyane, N., Salemme, R., Urquizar, C., Cotti, J., Guillaume, A., Vercher, J. L. et al. (2007). Oculomotor plasticity: are mechanisms of adaptation for reactive and voluntary saccades separate? Brain Research, 1135(1), 107-121.

Albano, J. E. (1996). Adaptive changes in saccade amplitude: oculocentric or orbitocentric mapping? Vision Research, 36(14), 2087-2098.

Catz, N., Dicke, P. W., \& Thier, P. (2008). Cerebellar-dependent motor learning is based on pruning a Purkinje cell population response. Proceedings of the National Academy of Sciences of the United States of America, 105(20), 7309-7314.

Collins, T. (2010). Extraretinal signal metrics in multiple-saccade sequences. Journal of Vision, 10(14), 7 .

Collins, T., \& Dore-Mazars, K. (2006). Eye movement signals influence perception: Evidence from the adaptation of reactive and volitional saccades. Vision Research, 46(21), 3659-3673.
Cotti, J., Guillaume, A., Alahyane, N., Pelisson, D., \& Vercher, J. L. (2007). Adaptation of voluntary saccades, but not of reactive saccades, transfers to hand pointing movements. Journal of Neurophysiology, 98(2), 602-612.

Cotti, J., Panouilleres, M., Munoz, D. P., Vercher, J. L., Pelisson, D., \& Guillaume, A. (2009). Adaptation of reactive and voluntary saccades: different patterns of adaptation revealed in the antisaccade task. Journal of Physiology, 587(1), 127-138.

Deubel, H. (1995). Separate adaptive mechanisms for the control of reactive and volitional saccadic eye movements. Vision Research, 35(23-24), 3529-3540.

Deubel, H., Wolf, W., \& Hauske, G. (1986). Adaptive gain control of saccadic eye movements. Human Neurobiology, 5(4), 245253.

Dore-Mazars, K., Vergilino-Perez, D., Collins, T., Bohacova, K., \& Beauvillain, C. (2006). The use of recurrent signals about adaptation for subsequent saccade programming depends on object structure. Brain Research, 1113(1), 153162.

Erkelens, C. J., \& Hulleman, J. (1993). Selective adaptation of internally triggered saccades made to visual targets. Experimental Brain Research, 93(1), 157-164.

Ethier, V., Zee, D. S., \& Shadmehr, R. (2008). Changes in control of saccades during gain adaptation. Journal of Neuroscience, 28(51), 13929-13937.

Frens, M. A., \& van Opstal, A. J. (1994). Transfer of short-term adaptation in human saccadic eye movements. Experimental Brain Research, 100, 293-306.

Fujita, M., Amagai, A., Minakawa, F., \& Aoki, M. (2002). Selective and delay adaptation of human saccades. Cognitive Brain Research, 13(1), 4152.

Gaymard, B., Rivaud, S., \& Pierrot-Deseilligny, C. (1994). Impairment of extraretinal eye position signals after central thalamic lesions in humans. Experimental Brain Research, 102(1), 1-9.

Golla, H., Tziridis, K., Haarmeier, T., Catz, N., Barash, S., \& Thier, P. (2008). Reduced saccadic resilience and impaired saccadic adaptation due to cerebellar disease. European Journal of Neuroscience, 27(1), 132-144.

Guthrie, B. L., Porter, J. D., \& Sparks, D. L. (1983). Corollary discharge provides accurate eye position information to the oculomotor system. Science, 221(4616), 1193-1195. 
Hallett, P. E., \& Lightstone, A. D. (1976). Saccadic eye movements to flashed targets. Vision Research, 16(1), 107-114.

Hernandez, T. D., Levitan, C. A., Banks, M. S., \& Schor, C. M. (2008). How does saccade adaptation affect visual perception? Journal of Vision, 8(8), 3-16.

Hopp, J. J., \& Fuchs, A. F. (2002). Investigating the site of human saccadic adaptation with express and targeting saccades. Experimental Brain Research, 144(4), 538-548.

Iwamoto, Y., \& Kaku, Y. (2010). Saccade adaptation as a model of learning in voluntary movements. Experimental Brain Research, 204(2), 145-162.

Keller, E. L. (1981). Brain stem mechanisms in saccadic control. In A.F. Fuchs \& W. Becker (Eds.), Progress in oculomotor research (pp. 57-62).

Kojima, Y., Iwamoto, Y., \& Yoshida, K. (2004). Memory of learning facilitates saccadic adaptation in the monkey. Journal of Neuroscience, 24(34), 7531-7539.

Lewis, R. F., Zee, D. S., Hayman, M. R., \& Tamargo, R. J. (2001). Oculomotor function in the rhesus monkey after deafferentation of the extraocular muscles. Experimental Brain Research, 141(3), 349-358.

Mays, L. E., \& Sparks, D. L. (1980). Saccades are spatially, not retinocentrically, coded. Science, 208(4448), 1163-1165.

McLaughlin, S. C. (1967). Parametric adjustment in saccadic eye movements. Perception \& Psychophysics, 2, 359-362.

Miller, J. M., Anstis, T., \& Templeton, W. B. (1981). Saccadic plasticity: parametric adaptative control feedback. Journal of Experimental Psychology: Human Perception and Performance, 7(2), 356-366.

Moschovakis, A. K., Scudder, C. A., \& Highstein, S. M. (1996). The microscopic anatomy and physiology of the mammalian saccadic system. Progress in Neurobiology, 50(2-3), 133-254.

Noto, C. T., Watanabe, S., \& Fuchs, A. F. (1999). Characteristics of Simian adaptation fields produced by behavioral changes in saccadic gain and direction. Journal of Neurophysiology, 81(6), 2798-2813.

Ostendorf, F., Liebermann, D., \& Ploner, C. J. (2010). Human thalamus contributes to perceptual stability across eye movements. Proceedings of the National Academy of Sciences of the United States of America, 107(3), 1229-1234.
Panouilleres, M., Neggers, S. F. W., Gutteling, T. P., Salemme, R., van der Stigchel, S., van der Geest, J. N. et al. (2011). Transcranial magnetic stimulation and motor plasticity in Human lateral cerebellum: dual effect on saccadic adaptation. Human Brain Mapping, in press.

Panouilleres, M., Urquizar, C., Salemme, R., \& Pelisson, D. (2011). Sensory Processing of Motor Inaccuracy Depends on Previously Performed Movement and on Subsequent Motor Corrections: A Study of the Saccadic System. PLoS ONE, 6(2), e17329.

Panouilleres, M., Weiss, T., Urquizar, C., Salemme, R., Munoz, D. P., \& Pelisson, D. (2009). Behavioral evidence of separate adaptation mechanisms controlling saccade amplitude lengthening and shortening. Journal of Neurophysiology, 101(3), 1550-1559.

Pelisson, D., Alahyane, N., Panouilleres, M., \& Tilikete, C. (2010). Sensorimotor adaptation of saccadic eye movements. Neuroscience and Biobehavioral Reviews, 34(8), 1103-1120.

Pisella, L., Alahyane, N., Blangero, A., Thery, F., Blanc, S., \& Pelisson, D. (2011). Righthemispheric dominance for visual remapping in humans. Philosophical Transactions of the Royal Society of London. Series B, Biological Sciences, 366(1564), 572-585.

Robinson, D. A. (1975). Oculomotor control signals. In G. Lennerstrand \& P. Bach-Y-Rita (Eds.), Basic Mechanisms of Ocular Motility and their clinical implications (pp. 337-378).

Schiller, P. H., \& Sandell, J. H. (1983). Interactions between visually and electrically elicited saccades before and after superior colliculus and frontal eye field ablations in the rhesus monkey. Experimental Brain Research, 49(3), 381-392.

Semmlow, J. L., Gauthier, G. M., \& Vercher, J. L. (1989). Mechanisms of short-term saccadic adaptation. Journal of Experimental Psychology: Human Perception and Performance, 15(2), 249-258.

Sommer, M. A., \& Wurtz, R. H. (2008). Brain circuits for the internal monitoring of movements. Annual Review of Neuroscience, $31,317-338$.

Sparks, D. L., \& Mays, L. E. (1983). Spatial localization of saccade targets. I. Compensation for stimulation-induced perturbations in eye position. Journal of Neurophysiology, 49(1), 4563.

Sparks, D. L., Mays, L. E., \& Porter, J. D. (1987). Eye movements induced by pontine stimulation: interaction with visually triggered saccades. Journal of Neurophysiology, 58(2), 300-318. 
Steinbach, M. J. (1987). Proprioceptive knowledge of eye position. Vision Research, 27(10), 17371744.

Straube, A., \& Deubel, H. (1995). Rapid gain adaptation affects the dynamics of saccadic eye movements in humans. Vision Research, 35(2324), 3451-3458.

Straube, A., Fuchs, A. F., Usher, S., \& Robinson, F. R. (1997). Characteristics of saccadic gain adaptation in Rhesus macaques. Journal of Neurophysiology, 77(2), 874-895.

Tanaka, M. (2003). Contribution of signals downstream from adaptation to saccade programming. Journal of Neurophysiology, 90(3), 2080-2086.

Wurtz, R. H. (2008). Neuronal mechanisms of visual stability. Vision Research, 48(20), 20702089.

Zimmermann, E., \& Lappe, M. (2009). Mislocalization of flashed and stationary visual stimuli after adaptation of reactive and scanning saccades. Journal of Neuroscience, 29(35), $11055-11064$

Zimmermann, E., \& Lappe, M. (2010). Motor signals in visual localization. Journal of Vision, 10(6), 1-11. 